LEADING ARTICLE

\title{
Fatigue in chronic cholestasis
}

\author{
P Milkiewicz, E J Heathcote
}

Fatigue is probably the most intriguing symptom affecting patients with chronic cholestatic disorders, in particular those with primary biliary cirrhosis. It is postulated that fatigue in patients with primary biliary cirrhosis may be associated with morphological abnormalities of the central nervous system secondary to accumulation of manganese. However, we are still far from understanding this complex issue.

See end of article for authors' affiliations

.....................

Correspondence to: Dr E J Heathcote, Toronto Western Hospital, University Health 'Network, 399 Bathurst St, 6B Fell Pavilon, Room 172

Toronto, Ontario M5T 2S8, Canada; jenny. heathcote@utoronto.ca

Accepted for publication 7 August 2003
S tedman's Medical Dictionary defines fatigue or bodily activity, characterized by a lessened capacity for work and reduced efficiency of accomplishment, usually accompanied by a feeling of weariness, sleepiness or irritability". ${ }^{1}$ This definition does not adequately describe the symptom of fatigue observed in several chronic diseases where the fatigue is not related to exercise and neither is it improved by rest. Fatigue under these circumstances generates severe distress and disability which is frequently out of proportion to the patient's medical status. Forty per cent of patients with multiple sclerosis describe fatigue as the single most disabling symptom and, somehow unexpectedly, find it more debilitating than weakness, spasticity, or even bowel or bladder dysfunction. ${ }^{2}$ Fatigue may also occur in patients with systemic lupus erythematosus (SLE) or rheumatoid arthritis. In SLE it correlates with depression but not with inflammatory or immunological markers of the disease. $^{3}$

Fatigue is probably the most intriguing symptom affecting patients with chronic cholestatic disorders. Neglected and overlooked for many years it has emerged over the last decade as an important clinical entity, in particular in patients with primary biliary cirrhosis (PBC). ${ }^{4}$ Despite the fact that many patients consider fatigue as "the most disabling symptom" it has not been properly analysed in the majority of therapeutic studies on patients with PBC. Several reports suggest that fatigue may affect between $70 \%$ and $85 \%$ of patients with $\mathrm{PBC}^{5}{ }^{6}$ although these numbers have been recently questioned. ${ }^{7}$

What makes fatigue in cholestatic disease such a fascinating issue is that it does not show any correlation with several markers of liver disease severity, including duration of disease, presence of pruritus, impairment of routine liver function tests, quantitative tests (aminopyrine breath test), Mayo risk score, or cognitive function. ${ }^{6} 8$ It is also not related to the patient's age or thyroid status. It does however correlate with sleep disturbance estimated by Pittsburgh sleep as "that state, following a period of mental
Gut 2004;53:475-477. doi: 10.1136/gut.2003.025155

quality index and depression assessed with the HDRS (Hamilton depression rating scale) or self related CES-D (Center for Epidemiological Studies-Depression scale).$^{6-8}$

Various tools have been used in the assessment of fatigue in patients with liver diseases. Early studies have estimated fatigue in a dichotomous way (present or absent) or according to the Guttman scale (absent, mild, moderate, or severe). ${ }^{9}$ Recent works have applied more advanced scales such as the fatigue severity score (which is a component of the fatigue activity index ) supported by visual analogue scales. ${ }^{6-8}$ This approach has led to more reliable estimation of this subjective symptom and its correlation with other symptoms of liver disease.

\section{"Central rather than peripheral mechanisms mediate fatigue"}

The aetiology of fatigue in individuals with liver disease remains a puzzle and its comparison with an enigma seems to be fully justified. ${ }^{4}$ There is increasing evidence that this phenomenon may be mediated centrally. Animal studies in the bile duct ligated rat model have shown aberrant central neurotransmission involving 5- $\mathrm{HT}_{1 \mathrm{~A}}$ receptors to be present and administration of a serotonin receptor agonist can improve fatigue in a "swim test" model. ${ }^{10}$ Also, small doses of interleukin $1 \beta$ (a cytokine involved in "sickness behaviours") were found to cause a significant decrease in locomotor function in acutely cholestatic rats. In the same model, altered function of the hypothalamic-pituitary-adrenal axis was shown to correlate with abnormal behaviour. ${ }^{11}{ }^{12}$ These findings support the hypothesis, at least in the model of acute cholestasis in rats, that central rather than peripheral mechanisms mediate fatigue. Nevertheless, studies on fatigue in the rat model should be interpreted with caution as models of acutely induced cholestasis in animals are far from ideal for the study of chronic cholestasis.

Impaired biliary excretion which occurs in chronic cholestasis may predispose to accumulation of several compounds, including heavy metals. In this issue of Gut, Forton and colleagues ${ }^{13}$ postulate that fatigue in patients with PBC may be associated with morphological abnormalities

Abbreviations: SLE, systemic lupus erythematosus; PBC, primary biliary cirrhosis; $M n$, manganese; ${ }^{1} \mathrm{H}$ MRS, proton magnetic resonance spectroscopy; MTR, magnetisation transfer ratio; PSE, portosystemic encephalopathy; CNS, central nervous system; GP, globus pallidus 
of the central nervous system (CNS), secondary to accumulation of manganese $(\mathrm{Mn})$ [see page 587]. As effective treatment of fatigue does not exist, the hypothesis that fatigue is a result of increased accumulation of $\mathrm{Mn}$ is very attractive in terms of potential application of new therapeutic modalities, including chelating agents. Mn is a neurotoxic heavy metal with increased affinity to dopaminergic neurones. In their study, Forton and colleagues ${ }^{13}$ apply sophisticated brain imaging technologies, including proton magnetic resonance spectroscopy $\left({ }^{1} \mathrm{H}\right.$ MRS) and magnetisation transfer ratio (MTR). ${ }^{1} \mathrm{H}$ MRS can provide helpful information about cerebral metabolism, and abnormal ${ }^{1} \mathrm{H}$ MRS patterns have been found to correlate with severity of hepatic encephalopathy. ${ }^{14}$ In this study, there was no difference in MRS choline/creatinine ratios between stage I-II PBC and healthy controls, suggesting an absence of even subclinical portosystemic encephalopathy (PSE) in these subjects to explain their fatigue. MTR is considered to be superior to conventional magnetic resonance imaging in detecting parenchymal abnormalities in the CNS as it incorporates a measurement of the exchange of protons between water and solid constituents of brain tissue. It has been demonstrated that patients with minimal hepatic encephalopathy have a reduced MTR, possibly due to increased brain water. ${ }^{15}$ The major findings of the study by Forton and colleagues ${ }^{13}$ is that globus pallidus (GP) MTR is significantly reduced in stage I and II patients with PBC compared with healthy controls. As this phenomenon can be secondary to accumulation of paramagnetic substances, the authors measured plasma Mn levels and found that the number of points scored in the fatigue impact score (both in cirrhotic and non-cirrhotic patients) correlated with blood $\mathrm{Mn}$ levels. The authors hypothesise that accumulation of $\mathrm{Mn}$ in the GP may be responsible for fatigue in the early stages of $\mathrm{PBC}$ in whom they showed no evidence of changes comparable with PSE on MRS.

\section{"Fatigue in patients with PBC may be associated with morphological abnormalities of the CNS, secondary to accumulation of manganese"}

This study shows how the aetiology of fatigue identified by validated questionnaires can be investigated using more scientifically sophisticated measure by involving the newest imaging technologies. However, these results have to be interpreted with caution. It is not clear what was the rationale for normalising the results against frontal white matter and the putamen but not, for example, against the caudate lobe and thalamus. In none of these four regions is Mn supposed to be deposited, and MTRs were in fact measured in all of these areas. Clearly, the easiest way to interpret the findings would be to analyse the raw MTR data. The authors have introduced indexes which cloud the picture and makes the paper difficult to follow in places. For example, the correlation between MTR and blood Mn in one of the control regions (putamen normalised to white matter) is nearly significant. Thus when it is used as the normalisation control region for GP, it is likely that some of the significant correlation is due to contamination by an association in the control. Additionally, very surprisingly, an autocorrelation (the normalised putamen against the normalised putamen) in table 2 is -0.58 and not 1 . Also, details of how the authors separated regions of interest are not provided (hand traced on individual patients? Standard $\mathrm{n} \times \mathrm{n} \times \mathrm{n}$ voxel placed in the region?). This information would be useful for researchers who would like to replicate and extend these findings. The authors are therefore right in saying that their results can only be considered as preliminary observations. The number of patients included was small (only four patients with grade III-IV PBC). It is a pity that the authors assumed that ${ }^{1} \mathrm{H}$ MRS would be abnormal in their subjects with stage III-IV PBC and did not measure it. It is important to clarify whether there truly is a clear distinction between groups because histological staging was presumably based on a single biopsy performed within a year before inclusion into the study. However, the value of staging of liver histology in $\mathrm{PBC}$ is limited as sampling error is common. Thus a comparison of ${ }^{1} \mathrm{H}$ MRS between stages I-II and III-IV PBC would have been well worth examining. This is also important because when results for MTR are calculated as indices normalised to white matter (GP/WM index), the difference between stages I-II and III-IV does not reach statistical significance.

Only five of 18 studied patients had elevated Mn blood levels. All four patients with stage III or IV PBC included in the study had normal Mn levels but half of them had fatigue. This suggests that only a minority of patients may be predisposed to impaired excretion/increased brain accumulation of $\mathrm{Mn}$ and severity of liver fibrosis correlated with neither $\mathrm{Mn}$ accumulation nor fatigue. A lack of correlation between stage of liver histology in PBC and fatigue has previously been shown by Huet et al who in a large study involving 116 patients showed that fatigue severity did not correlate with stage of liver disease when patients with grade I-II PBC were compared with patients with grade III-IV. ${ }^{6}$ But it is the degree of bile duct loss and not liver fibrosis which governs the severity of cholestasis and it is the former that would promote retention of Mn. There may be a discordance between these two histological features in $\mathrm{PBC}^{16}$ - this has not been addressed by the authors of this paper. In both groups of patients with $\mathrm{PBC}$ in this study, bilirubin levels were normal, and other biochemical markers of cholestasis were not mentioned. Pruritus, but only if it was nocturnal, was an exclusion criterion!

\section{"Further studies in larger cohorts of patients, in particular in those in whom fatigue resolved after liver transplanta- tion, are required"}

Is there a selective defect in excretion of Mn in PBC and could this retention of $\mathrm{Mn}$ be a cause of fatigue in all patients with PBC? On the basis of this and other studies the answer is certainly no. As rightly pointed out by the authors, further studies in larger cohorts of patients, in particular in those in whom fatigue resolved after liver transplantation, are required.

This study is a step in a right direction but also shows that we are still far from understanding the complex issue of fatigue in chronic cholestasis which is so frustrating, particularly for the patient but also for the treating physician

\section{Authors' affiliations}

P Milkiewicz, Department of Gastroenterology, Pomeranian Medical School, 71-242 Szczecin, Unii Lubelskiej 1, Poland, and Toronto Western Hospital, University Health Network, Toronto, Ontario, Canada E J Heathcote, Toronto Western Hospital, University Health Network, Toronto, Ontario, Canada

\section{REFERENCES}

1 Stedman's Medical Dictionary, 25 edn. Baltimore: Williams \& Wilkins, 1990. 2 Bakshi R. Fatigue associated with multiple sclerosis: diagnosis, impact and management. Mult Scler 2003;9:219-27.

3 Omdal R, Waterloo K, Koldingsnes W, et al. Fatigue in patients with systemic lupus erythematosus: the psychosocial aspects. J Rheumatol 2003;30:283-7.

4 Jones EA. Fatigue associated with chronic liver disease: a riddle wrapped in a mystery inside an enigma. Hepatology 1995;22:1606-8.

5 Witt-Sullivan $\mathrm{H}$, Heathcote EJL, Cauch K, et al. The demography of primary biliary cirrhosis in Ontario, Canada. Hepatology 1990;12:98-105. 
6 Huet PM Deslauries J Tran A, et al. Impact of fatigue in the quality of life of patients with primary biliary cirrhosis. Am J Gastroenterol 2000;95:760-7.

7 Goldblatt J, Taylor PJS, Lipman T, et al. The true impact of fatigue in primary biliary cirrhosis: a population study. Gastroenterology 2002;122:1235-41.

8 Cauch-Dudek K, Abbey D, Steward DE, et al. Fatigue in primary biliary cirrhosis. Gut 1998:43:705-10.

9 Prince MI, James OFW, Holland NP, et al. Validation of a fatigue impact score in primary biliary cirrhosis: towards a standard for clinical and trial use. $J$ Hepato 2000;32:368-73.

10 Swain MG, Maric M. Improvement in cholestasis-associated fatigue with serotonin receptor agonist using a novel rat model of fatigue assessment. Hepatology 1997;25:292-4.

11 Swain MG, Beck P, Rioux K, et al. Augmented interleukin-1 beta-induced depression of locomotor activity in cholestatic rats. Hepatology 1998;28:1561-5.
12 Swain MG, Maric M. Defective corticotropin-releasing hormone mediated neuroendocrine and behavioral responses in cholestatic rats: implications for cholestatic liver disease-related sickness behaviors. Hepatology 1995;22:1560-64.

13 Forton DM, Patel N, Prince $M$, et al. Fatigue and primary biliary cirrhosis: association of globus pallidus magnetisation transfer ratio measurements with fatigue severity and blood manganese levels. Gut 2004;53:587-92.

14 Butterworth RF. Pathogenesis of hepatic encephalopathy: new insight from neuroimaging and molecular studies. J Hepatol 2003;39:278-85.

15 Cordoba J, Alonso J, Rovira A, et al. The development of low-grade cerebral oedema in cirrhosis is supported by the evolution of (1) $\mathrm{H}$-magnetic resonance abnormalities after liver transplantation. J Hepatol 2001;35:598-604.

16 Vleggaar FP, van Buuren HR, Zondervan PE, et al. Jaundice in non-cirrhotic primary biliary cirrhosis: the premature ductopenic variant. Gut 2001;49:276-81.

\section{GI SNAPSHOT}

\section{Management of swallowed razor blades - retrieve or wait and see?}

\section{Question}

A 16 year old boy with a long history of self harm was admitted for the third time in four weeks with a history of ingestion of a number of shaving blades (fig 1). On previous occasions, endoscopic intervention with the use of an overtube under general anaesthesia had been successful in their safe retrieval. However, on the third occasion, a delay to endoscopy of 36 hours (due to a combination of late presentation and lack of access to the operating theatre) allowed the blades to progress beyond the pylorus into the small bowel, beyond the reach of a standard upper gastrointestinal endoscope (fig 2).

How should this young man now be managed?

- Push enteroscopy with the use of an overtube and removal of the blades?

- Laparotomy and surgical removal of the blades?

- Conservative management?

See page 486 for answer

J R Butterworth, K Wright, R A Boulton, S Pathmakanthan, J Goh Gastrointestinal Unit, University Hospital of Birmingham NHS Trust, Queen Elizabeth and Selly Oak Hospitals, Raddllebarn Rd, Birmingham B15, UK

\section{Correspondence to: Dr J R Butterworth, Royal Shrewsbury Hospital, Mytton Oak Rd,} Shrewsbury SY3 8XQ, UK; j.r.butterworth@btinternet.com

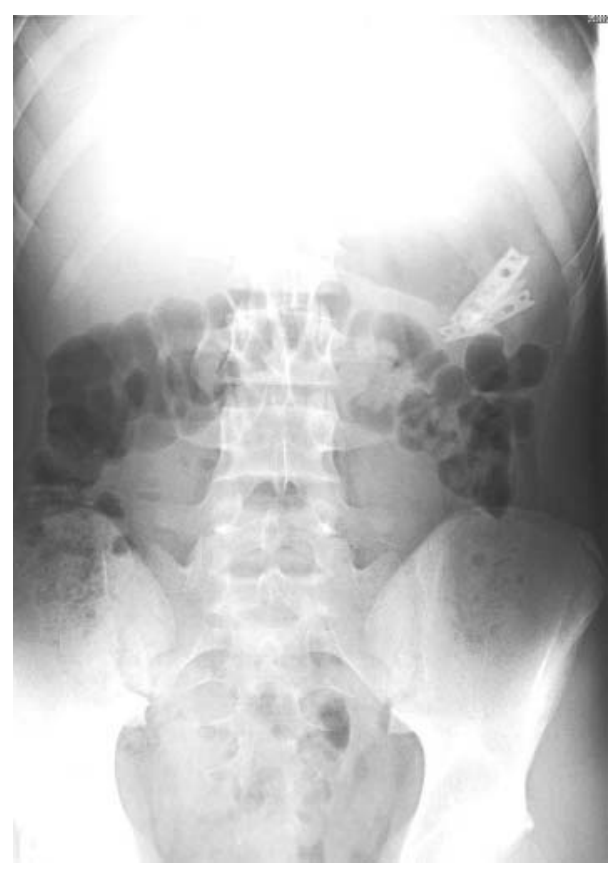

Figure $1 X$ ray showing the razor blades in the stomach.

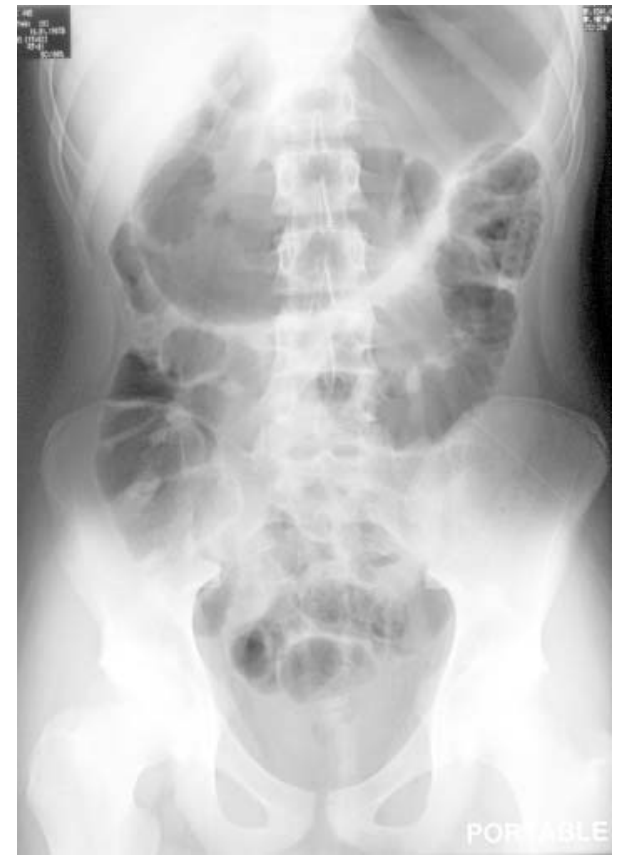

Figure $2 X$ ray showing the razor blades in the small bowel, beyond the reach of a standard upper gastrointestinal endoscope. 